\title{
Changing trends of dengue disease: a brief report from a tertiary care hospital in New Delhi
}

\section{Authors}

Monika Matlani ${ }^{1}$

Anita Chakravarti

${ }^{1} \mathrm{MD}$, Senior Resident ${ }^{2} \mathrm{MD}$, Director Professor Microbiology, Maulana Azad Medical College New Delhi, India
Submitted on: 11/10/2010 Approved on: 11/13/2010

Correspondence to: Dr. Anita Chakravarti Director Professor Microbiology

Maulana Azad Medical College

New Delhi, India anita.chakravarti@yahoo.com

Financial Support: Department of Science and Technology, New Delhi

We declare no conflict of interest.
Dear Editor,

this is in response to the paper "Correlation of disease spectrum among four Dengue serotypes: a five-year hospital based study from India", published by Dr Rajni Kumaria in the Journal (vol.14, no. 2). ${ }^{1}$ This study was conducted in the virology laboratory of Maulana Azad Medical College, New Delhi, from January 2002 to November 2006 as a part of a project funded by the Indian Council of Medical Research (ICMR). The findings obtained from the same laboratory for the subsequent three years (2007 -2009) were relatively different from the earlier published one ${ }^{1}$ and are being reported here. We wish to highlight the changing trends in the presentation of dengue from the same Dengue Serosurveillance Laboratory during this period.

In contrast to 2002-2006 findings where Den-2 was the commonest serotype reported, ongoing study observed Den-1 to be the most prevalent serotype during 2007-2009. Den-1 was isolated in $50 \%$ of RT-PCR positive cases (20/40), whereas Den-2, Den-3 and Den-4 were identified in $22.5 \%$ (9/40), $15 \%(6 / 40)$ and $12.5 \%(5 / 40)$ of cases respectively, indicating the replacement of earlier circulating Den-2 and Den- 3 by Den- 1 . Maximum number of Den-1 cases were isolated in the year 2008 (findings published earlier). ${ }^{2}$ These findings provide an insight to the changing trend of dengue serotypes indicating that all four serotypes are continuously co-circulating, which makes this region prone to epidemic spreads.

Although the abovementioned study ${ }^{1}$ and few other studies ${ }^{3}$ have reported Den-1 to be associated with a milder form of disease, our study demonstrates a rise in number of Den-1 affected severe cases during 20072009. Sixty percent of the Den-1 affected cases presented with severe DHF. Moreo- ver incidence of arthralgia and myalgia was also observed in significantly higher number of Den-1 cases. This increase in DHF cases due to Den-1 could have been due to heterologous infection leading to ADE (antibodydependant enhancement) resulting in sss imbalance of the levels of cytokines, or due to some unknown genetic changes in the circulating strain which could have led to increased virulence of the particular strain. Various studies have been done to establish correlation between causative serotype and disease outcome. Some of them have proposed that Den-2 and Den-3 may cause more severe disease, ${ }^{4,5}$ however, we observed that even Den-1 can present with severe form of disease. Few other researchers have also suggested that the DEN-1 strains were more pathogenic among children having primary infections. ${ }^{6}$ Our findings illustrate that each serotype has unique characteristics and can present with severe manifestations, in a particular population depending upon its interaction with the host response.

For establishing conclusive relationship between the serotypes and disease manifestations studies are further ongoing in our set up for identifying various virus and host factors responsible for severity of dengue disease. Such studies would help to predict future outbreaks, developing interventions to control dengue outbreaks, and developing an effective dengue vaccine.

\section{REFERENCES}

1. Kumaria R. Correlation of disease spectrum among four Dengue serotypes: a five years hospital based study from India Braz J Infect Dis 2010; 14(2):141-146.

2. Chakravarti A, Kumar A, Matlani M. Displacement of dengue virus type 3 and type 2 by dengue virus type 1 in Delhi during 2008. IJMM 2010; 28(4):412. 
3. Corwin AL, Larasati RP, Bangs MJ et al. Epidemic dengue transmission in southern Sumatra, Indonesia. Trans R Soc Trop Med Hyg 2001; 95:257-65.

4. Endy TP, Nisalak A, Chunsuttiwat $\mathrm{S}$ et al. Spatial and temporal circulation of dengue virus serotypes: a prospective study of primary school children in Kamphaeng Phet, Thailand. Am J Epidemiol 2002; 56:52-9.
5. Kalayanarooj S, Nimmannitya S. Clinical and laboratory presentations of dengue patients with different serotypes. WHODengue Bulletin. 2000; 24:53-9.

6. Nisalak A, Endy TP, Nimmanniya S et al. Serotype-specific dengue virus circulation and dengue disease in Bangkok, Thailand from 1973-1979. Am J Trop Med Hyg 2003; 68:191202. 